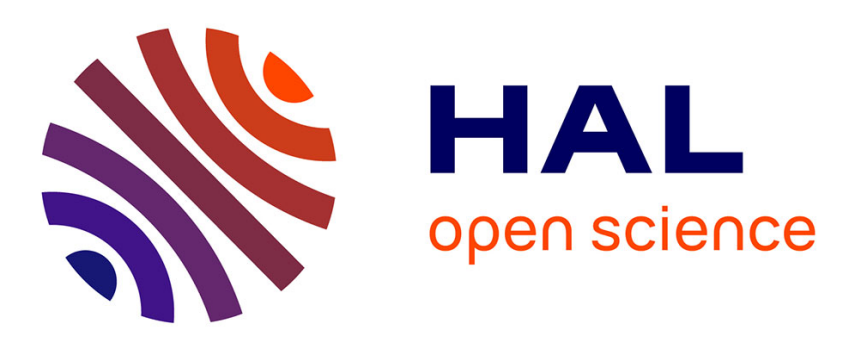

\title{
Combinatorial Designs and Hypergraphs of Diameter One
}

\author{
Jean-Claude Bermond, Johny Bond
}

\section{To cite this version:}

Jean-Claude Bermond, Johny Bond. Combinatorial Designs and Hypergraphs of Diameter One. Annals of the New York Academy of Sciences, 1989, 576 (1 Graph Theory), pp.51-62. 10.1111/j.17496632.1989.tb16382.x . hal-02493603

\section{HAL Id: hal-02493603 https://hal.science/hal-02493603}

Submitted on 27 Feb 2020

HAL is a multi-disciplinary open access archive for the deposit and dissemination of scientific research documents, whether they are published or not. The documents may come from teaching and research institutions in France or abroad, or from public or private research centers.
L'archive ouverte pluridisciplinaire HAL, est destinée au dépôt et à la diffusion de documents scientifiques de niveau recherche, publiés ou non, émanant des établissements d'enseignement et de recherche français ou étrangers, des laboratoires publics ou privés. 


\title{
COMBINATORIAL DESIGNS
}

\section{AND \\ HYPERGRAPHS OF DIAMETER ONE}

\author{
$J$-C. Bermond and J. Bond \\ * Laboratoire de Recherche en Informatique \\ U.A. 410 du C.N.R.S., Université de Paris Sud \\ F-91405 ORSAY Cedex
}

\begin{abstract}
The problem considered here is motivated by the study of combinatorial properties of interconnection networks, where the processors share a communication medium like a bus. It consists in finding the maximum number of vertices of a hypergraph of maximum degree $\Delta$, maximum edge-size $r$ and diameter $D$. Here we consider the case $D=1$, that is when each pair of vertices belongs to at least one edge. In [6], we solved the case $\Delta$ small and also the case $r \leq 3$. Here we solve completely the case $r=4$; we show that the maximum number of vertices is $3 \Delta$ for $\Delta \equiv 2$ or $3(\bmod 4)$ and $3 \Delta+1$ otherwise, thus answering a problem of [6]. This leads also to study new covering designs related to the work of C. Huang, E. Mendelsohn and A. Rosa [11]. In particular we prove that there exists a decomposition of $K_{v}$ into a $K_{10}$ and $K_{4}$ 's if and only if $v \equiv 7$ or $10(\bmod 12), v \neq 7,19,22$.
\end{abstract}

* research partially supported by P.R.C. Math Info 


\section{Introduction}

Recent advances in technology, especially the advent of very large scale integrated (VLSI) circuit technology have enabled very complex interconnection networks to be constructed. Thus it is of great interest to study the topologies of interconnection networks, and, in particular, their associated graphical properties. Usually point to point connections are considered and the computer network is modeled by a graph in which the nodes or vertices correspond to the computer centers in the network and the edges correspond to the communication links. Surveys on classical interconnection networks can be found in $[4,7,9]$.

Here we consider the case in which the computers share a communication medium such as a bus; the network is modeled by a hypergraph, where the nodes correspond to the computer centers and the (hyper)edges to the buses.

Although these networks are now less used than the classical ones with point-topoint connections, their importance is growing and they might be very important in the near future. Our interest in this subject came from discussions with persons working at the French National Telecommunication Center (CNET), on a project called REBUS.

One important measure of the power of an interconnection network is the length of the longest path that the messages must travel from one node to another in the network, i.e. the distance between the nodes. It is advantageous to make these distances as small as possible, since this will reduce the message delay and also the message traffic density in the links. Worst case distance corresponds to the diameter of the associated graph or hypergraph. Similarly other network characteristics correspond to parameters of graphs or hypergraphs, e.g. mean distance, symmetry, connectivity, ...

Furthermore physical limitations usually restrict the number of connections for each processor; for bus connection networks it means that a node must not belong to too many buses or equivalently that each vertex has a bounded degree.

For bus connection networks one more constraint is to be considered: the traffic load on each bus is limited. Since, for general networks without symmetry properties, the traffic per bus is not easily calculated, we will restrict our study to networks with some uniformity and, to a first approximation, replace the constraint of traffic load by the limitation of the number of processors connected to each bus. This means that the size $r$ of the edges of the associated hypergraph is bounded.

It is easy to see that it is impossible to optimize all the criteria at the same time: a comparison of some different criteria on a few structures has been done by Wittie [14]; see also [5]. Here we consider the extremal problem of determining the 
maximum number of vertices of a bus network with given maximal degree, maximal bus-size and diameter. We restrict our attention to the case of diameter one, pursuing the study initiated in [6]. For other cases in particular $\Delta=2$ and references on bus networks see [5].

\section{Notation and definitions}

We shall follow the terminology of Berge's book [3].

- $H=(X, E)$ will denote a hypergraph with vertex set $X$ and edge set $E$

- the degree of a vertex is the number of edges containing it

- the maximum degree will be denoted by $\Delta$

- the maximal size of an edge will be denoted by $r$

- if all the edges have size $r$, the hypergraph is said $r$-uniform (also sometimes called $r$-graph in the literature)

- a path connecting $x$ and $y$ in a hypergraph is a sequence $x=x_{1}, E_{1}, x_{2}, \cdots, x_{i}, E_{i}, x_{i+1}, \cdots, E_{p}, x_{p+1}=y$ with $\left\{x_{i}, x_{i+1}\right\} \subseteq E_{i}$ for $1 \leq i \leq p$.

- the length of a path will be the number of its edges

- the distance between two vertices is the length of a shortest path between them

- the diameter of the hypergraph is the maximum of the distances over all pair of vertices.

Therefore, a hypergraph is of diameter 1 if and only if any pair of vertices belongs to at least one edge.

\section{The $(\Delta, 1, \mathbf{r})$-hypergraph problem}

Let us call a hypergraph of diameter $D$, maximum degree $\Delta$ and maximum size of edges $r$, a $(\Delta, D, r)$-hypergraph. The problem considered in the introduction consists in determining the maximum number $n(\Delta, D, r)$ of vertices of a $(\Delta, D, r)$-hypergraph. Here we deal with the case $D=1$.

Let us recall first some results of [6]:

Proposition 1: $n(\Delta, 1, r) \leq 1+\Delta(r-1)$.

Proposition 2: $n(\Delta, 1, r)=1+\Delta(r-1)$ if and only if there exists a $(v, r, 1)$-design with

$$
v=1+\Delta(r-1) \text {. }
$$


Proposition 3: $n(\Delta, 1,3)=2 \Delta+1$ if $\Delta \equiv 0$ or $1(\bmod 3)$

and

$n(\Delta, 1,3)=2 \Delta$ if $\Delta \equiv 2(\bmod 3)$.

Proposition 4: (i) $n(\Delta, 1,4)=3 \Delta+1$ if $\Delta \equiv 0$ or $1(\bmod 4)$

(ii) $n(\Delta, 1,4)=3 \Delta$ if $\Delta \equiv 2(\bmod 4)$

(iii) $n(\Delta, 1,4)=3 \Delta$ or $3 \Delta-1$ if $\Delta \equiv 3(\bmod 4)$

It is easy to prove that $n(3,1,4)=8$ which is $3 \Delta-1$. In [6], we conjectured that $n(\Delta, 1,4)=3 \Delta$ for $\Delta \equiv 3(\bmod 4), \Delta>3$. The original aim of this article was to prove this conjecture. Different tentatives to solve it gave rise to some new problems in design theory, which are interesting in themselves.

Main Theorem $: n(\Delta, 1,4)=3 \Delta$ if $\Delta \equiv 3(\bmod 4), \Delta>3$.

\section{Notations of design theory}

We use the classical notations in design theory [10]. Let $K$ be a set of integers, $\lambda$ and $v$ be integers. A pairwise balanced design $B(K, \lambda, v)$ is a collection of subsets, called blocks, of a given set on $v$ vertices, such that the size of each block belongs to $K$ and each pair of vertices belongs to exactly $\lambda$ blocks. In what follows, we will always consider the case $\lambda=1$ and we will denote a $B(K, 1, v)$ shortly by $B(K, v)$. A $B(K, v)$ design can be viewed as a partition of the edges of the complete graph on $v$ vertices into complete subgraphs, each of size belonging to $K$. A $B(K, v)$ can also be considered as a hypergraph with vertex set of cardinality $v$, edges of size belonging to $K$ and of diameter one.

We will denote by $B(K)$ the set of values of $v$ for which there exists a $B(K, v)$ design. For example $B(3)$ is the set of values for which there exists a Steiner triple system and it is well known that $B(3)=\{v \mid v \equiv 1$ or $3(\bmod 6)\}$. It is also known that $B(4)=\{v \mid v \equiv 1$ or $4(\bmod 12)\}$. When all the blocks have the same size $k$ except one which has size $m$, we will write $B\left(\left\{k, m^{*}\right\}, v\right)$ and $B\left(\left\{k, m^{*}\right\}\right)$ (the star meaning that there exists exactly one block of size $m$ ). For example Brouwer's theorem in [8] states that $B\left(4,7^{*}\right)=\{v \mid v \equiv 7$ or $10(\bmod 12), v \neq 10,19\}$.

If furthermore the blocks can be partitioned into parallel classes (where a parallel class form a partition of the vertices), then the design is called resolvable and denoted by $R B(K, v)$ and the set of values of $v$ for which there exists a $R B(K, v)$ by $R B(K)$. For example in the case $K=\{3\}$ a $R B(3, v)$ is known as a Kirkman triple system and it has been proved by Ray-Chaudhuri and Wilson in [13], that $R B(3)=\{v \mid v \equiv 3(\bmod 6)\}$. 


\section{Short proof of the main theorem}

Main Theorem: $n(\Delta, 1,4)=3 \Delta$ if $\Delta \equiv 3(\bmod 4), \Delta>3$.

Proof: Let $\Delta=4 t+3$ and $n=3 \Delta=12 t+9$ with $t \geq 1$. Brouwer's theorem [8] shows that there exists a $B\left(\left\{4,7^{*}\right\}, v\right)$ for $v \equiv 7$ or $10(\bmod 12), v \neq 10,19$. In particular for $t \geq 1$, there exists a decomposition of $K_{12 t+10}$ into $K_{4}$ 's and exactly one $K_{7}$. Let $v_{1}, v_{2}, \cdots, v_{7}$ be the vertices of the $K_{7}$. Consider the hypergraph on $12 t+9$ vertices obtained by deleting the vertex $v_{7}$, the edges of the $K_{7}$ constructed on the vertices $v_{1}, v_{2}, \cdots, v_{7}$ and adding the three quadruples $\left\{v_{1}, v_{2}, v_{3}, v_{4}\right\},\left\{v_{1}, v_{2}, v_{5}, v_{6}\right\},\left\{v_{3}, v_{4}, v_{5}, v_{6}\right\}$; this hypergraph is clearly of diameter one, edge size at most 4 (all the $K_{4}$ 's containing $v_{7}$ are now $K_{3}$ 's) and the maximum degree is $4 t+3$. Indeed a vertex $v_{i}$ was in $4 t+1 K_{4}$ 's and a $K_{7}$, and therefore it belongs now to $4 t+3 K_{4}$ 's. Any other vertex was in $4 t+3$ $K_{4}$ 's and now it belongs to $4 t+2 K_{4}$ 's and one $K_{3}$.

\section{Structural properties}

Proposition: If there exists a $(\Delta, 1,4)$-hypergraph on $3 \Delta$ vertices then any vertex $x$ is of degree $\Delta$ and of one of the following kinds:

type a: $x$ belongs to exactly one $K_{3}$ and furthermore the intersection of any two edges containing it is reduced to this vertex.

type b: $x$ belongs only to $K_{4}$ 's and there exists a unique vertex $y$ such that the pair $\{x, y\}$ belongs to exactly two $K_{4}$ 's, otherwise for any other vertex $z$, the pair $\{x, z\}$ belongs to exactly one $K_{4}$.

Proof: Let $x$ be a given vertex. The diameter being 1, any vertex different from $x$ must belong to an edge containing $x$. Suppose $x$ belongs to $\delta_{i}$ edges of size $i(i=2,3,4)$. We have $\delta_{2}+\delta_{3}+\delta_{4} \leq \Delta$ and $n \leq 1+\delta_{2}+2 \delta_{3}+3 \delta_{4}$. If $\delta_{2}+\delta_{3}+\delta_{4}=d(x) \leq \Delta-1$, then $n \leq 3(\Delta-1)+1=3 \Delta-2$, a contradiction. Therefore every vertex is of degree $\Delta$. We cannot have $\delta_{2} \geq 1$, otherwise $n \leq 3(\Delta-1)+2=3 \Delta-1$. We cannot have $\delta_{3} \geq 2$, otherwise $n \leq 3(\Delta-2)+5=3 \Delta-1$. If $\delta_{3}=1$, then $n \leq 3 \Delta$ and the equality is possible if and only if all the edges containing $x$ are disjoint (except in $x$ ). If $\delta_{3}=0, n=3 \Delta$ implies that exactly two edges intersect in two vertices $x$ and $y$.

Let $n_{a}$ be the number of vertices of type a and $n_{b}$ those of type b. By the proposition above we have: 


$$
\begin{aligned}
& n_{a}+n_{b}=3 \Delta \\
& n_{a} \equiv 0(\bmod 3) \\
& n_{b} \equiv 0(\bmod 2)
\end{aligned}
$$

That implies $n_{b} \equiv 0(\bmod 6)$. Furthermore if $\Delta=4 t+3$ then $n_{a} \equiv 3(\bmod 6)$.

Note that $n_{b}=0$ is impossible, otherwise by adding a new vertex to all the $K_{3}$ 's (which are disjoint) that will give rise to a decomposition of $K_{12 t+10}$ into $K_{4}$ 's which does not exist. So the smallest possible value for $n_{b}$ is 6 and that was the key of the construction in the proof of the main theorem, where we have exactly six vertices of type b, i.e. $v_{1}, v_{2}, \cdots, v_{6}$. In what follows we consider what happens for different admissible values of $n_{a}$ and $n_{b}$.

\section{A construction for the case $\Delta \equiv 7(\bmod 12)$}

Here we propose a construction, for the case $\Delta \equiv 7(\bmod 12)$, in which the number of vertices of type $a$ and the number of vertices of type $b$ are increasing with $\Delta$.

Suppose $\Delta=12 t+7$, and we have $n=36 t+21$ vertices. We shall construct a $(12 t+7,1,4)$-hypergraph with $24 t+15$ vertices of type a and $12 t+6$ vertices of type b. As $24 t+15 \equiv 3(\bmod 6)$, there exists a Kirkman Triple System, $R B(3,24 t+15)$, covering our vertices of type a. Let $S_{i}, 1 \leq i \leq 12 t+7$ denote the parallel classes; each of them contains $8 t+5$ triples. Let $b_{i}, 1 \leq i \leq 12 t+6$ be the vertices of type b. To each triple of $S_{i}, 1 \leq i \leq 12 t+6$ we add the vertex $b_{i}$, therefore obtaining edges of size 4 . On the set of vertices of type $b$, of size $12 t+6$, we construct a $(4 t+2,1,4)$-hypergraph (by proposition 4-(ii)). In this way we obtain a hypergraph of diameter 1, maximal edge size 4. Each vertex of type a belongs to $12 t+7$ triplets in the Kirkman Triple System, and therefore is of degree $12 t+7$. A vertex $b_{i}$, belongs to $4 t+2$ edges of the hypergraph constructed on the vertices of type $\mathrm{b}$ and to $8 t+5$ quadruples obtained from the triples of the parallel class $S_{i}$. Its degree is also $12 t+7$.

\section{Possible extensions}

In the case $\Delta=4 t+3$, the smallest possible value for $n_{a}$ is 3 . If there exists a $(4 t+3,1,4)$-hypergraph containing 3 vertices of type a, then by deleting these vertices we obtain a decomposition of the edges of a $K_{12 t+6}$ plus a perfect matching (constructed on the vertices of type b) into $K_{4}$ 's and 3 parallel classes of $K_{3}$ 's. Conversely if there exist a decomposition of a $K_{12 t+6}$ plus a perfect matching into $K_{4}$ 's and 3 parallel classes of $K_{3}$ 's, we can construct easily a $(4 t+3,1,4)$-hypergraph on $12 t+9$ 
vertices by adding three vertices $v_{1}, v_{2}, v_{3}$, and adding them respectively to the triples of each class. Therefore another solution to our problem could be found if we can solve the following conjecture:

Conjecture ${ }^{*}: K_{12 t+6}$ plus a perfect matching can be decomposed into $K_{4}$ 's and three parallel classes of $K_{3}$.

More generally, suppose we have a decomposition of $K_{12 t+9}$ with $n_{a}$ vertices of type a and that the edges of the $K_{n_{a}}$ constructed on the $n_{a}$ vertices can be partitioned into $K_{4}$ 's and a parallel class of $K_{3}$. That can be done if and only if $n_{a} \equiv 0$ or $3(\bmod 12))$. Indeed if we add a vertex we must obtain a $B\left(4, n_{a}+1\right)$ design. Then, by deleting these $n_{a}$ vertices what remains is a decomposition of $K_{n_{b}}$ plus a perfect matching into $K_{4}$ 's and $n_{a}$ parallel classes of $K_{3}$ 's. Note that it implies that $2 n_{a} \leq n_{b}$. An interesting case is when $2 n_{a}=n_{b}$. If $n=12 t+9$ this implies $n_{a}=4 t+3$ and $n_{b}=8 t+6$, and with the constraint $n_{a} \equiv 3(\bmod 12)$ we have $t=3 k$. In this case we must have a decomposition of $K_{24 k+6}$ plus a perfect matching into parallel classes of $K_{3}$.

More generally we conjecture:

Conjecture: $K_{6 t}$ plus a perfect matching can be decomposed into parallel classes of $K_{3}(t \geq 3)$.

This problem is similar to the problem of decomposing $K_{6 t}$ minus a perfect matching into parallel classes of $K_{3}$, ("nearly Kirkman Triple systems", see the article of Baker and Wilson [2]).

Example for $6 t=18$ :

Let $X=\left(\mathbb{Z}_{9} \times \mathbb{Z}_{2}\right)$ then 9 parallel classes are:

$$
\begin{aligned}
C_{i}= & \{(i, 0),(i+1,0),(i+3,0)\} \cup\{(i+2,0),(i+7,0),(i+2,1)\} \cup\{(i+8,0),(i+1,1),(i+7,1)\} \cup \\
& \cup\{(i+4,0),(i, 1),(i+5,1)\} \cup\{(i+5,0),(i+6,1),(i+8,1)\} \cup\{(i+6,0),(i+3,1),(i+4,1)\} .
\end{aligned}
$$

The other cases give rise to the general problem.

Problem: For what values of $t$ and $m$ can $K_{6 t}$ plus a perfect matching be decomposed

* This conjecture have been quite completely solved by Assaf, Mendelsohn and Stinson [1] who proved it for $t>22$.

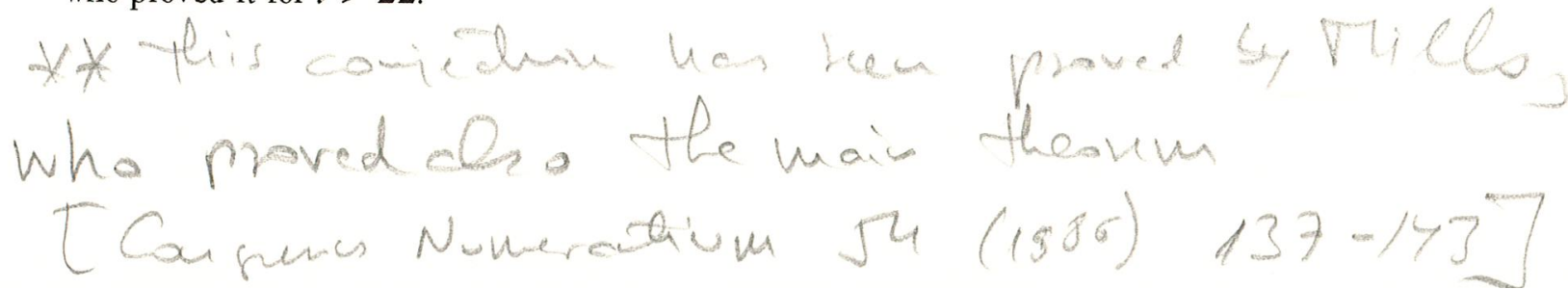


into $K_{4}$ 's and $m$ classes of $K_{3}$ (where $m \leq 3 t$ ).

The case $m=0$ follows from the fact that the edges of $K_{6 t}$ can be covered with $3 t^{2}$ quadruples (see Mills in[12]). In such a covering each vertex belongs exactly to $2 t$ $K_{4}$ 's, and therefore for each vertex there is exactly one edge covered twice. That is equivalent to say that $K_{6 t}$ plus a perfect matching can be decomposed into $K_{4}$ 's.

\section{Existence of $B\left(\left\{4,10^{*}\right\}, v\right)$}

Now let us fix $n_{b}=12 k+6$. Similarly as in the proof of the main theorem, we can obtain a $(4 t+3,1,4)$-hypergraph on $n=12 t+9$ vertices $(t \geq k)$, if we can decompose $K_{n+1}$ into a $K_{n_{b}+1}$ and $K_{4}$ 's; indeed as we have seen just above $K_{n_{b}}$ plus a perfect matching can itself be decomposed into $K_{4}$ 's. Let $m=n_{b}+1$, then this problem is equivalent to the existence of a $B\left(\left\{4, m^{*}\right\}, v\right)$ for $m=12 k+7$. More generally one can ask the following problem:

Problem: Determine $B\left\{4, m^{*}\right\}$ for $m \equiv 1(\bmod 3)$

Note that this problem was first considered by Huang, Mendelsohn and Rosa in [11]. In fact they defined a PRP 2-(3,4,v,m), as a partition of $K_{v}$ into $K_{4}$ 's and $m$ parallel classes of $K_{3}$. By adding $m$ points each joined to the triples of a parallel class from a PRP 2-(3,4,v,m) we obtain a $B\left(\left\{4, m^{*}\right\}, v+m\right)$ design.

Necessary conditions for the existence of a $B\left(\left\{4, m^{*}\right\}, v\right)$ are

$$
\begin{gathered}
v \equiv 1 \text { or } 4(\bmod 12) \text { if } m \equiv 1 \text { or } 4(\bmod 12) \\
v \equiv 7 \text { or } 10(\bmod 12) \text { if } m \equiv 7 \text { or } 10(\bmod 12) \\
\text { and if } v \neq m, v \geq 3 m+1 .
\end{gathered}
$$

Indeed the number of edges of $K_{v}-K_{m}$ must be a multiple of 6 , that is $v(v-1) \equiv m(m-1)(\bmod 12)$; furthermore the degree must be a multiple of 3 that is $v \equiv 1(\bmod 3)$. Finally as the existence of such a design corresponds to a decomposition of $K_{v-m}$ into $K_{4}$ 's and $m$ parallel classes of $K_{3}$ 's, the degree of any vertex of $K_{v-m}$ is at least $2 m$, that is $v-m-1 \geq 2 m$ or $v \geq 3 m+1$.

For $m=4$ the existence of a $B\left(\left\{4, m^{*}\right\}, v\right)$ corresponds to the existence of a $B(4, v)$ design. For $m=7$ Brouwer's theorem shows that these necessary conditions are sufficient, that is

$$
B\left(\left\{4,7^{*}\right\}\right)=\{v \mid v \equiv 7 \text { or } 10(\bmod 12) ; v \neq 10,19\} .
$$

Here we prove that they are also sufficient for $m=10$. 
Theorem: $B\left(\left\{4,10^{*}\right\}\right)=\{v \mid v \equiv 7$ or $10(\bmod 12) ; v \neq 7,19,22\}$.

Proof: We are looking for a decomposition of $K_{v}-K_{10}$ into $K_{4}$ 's or equivalently a decomposition of $K_{v-10}$ into $K_{4}$ 's and 10 parallel classes of $K_{3}$ 's. The proof proceeds exactly as Brouwer's one.

Lemma 1: If $3 t+m$ and $3 h+m \in B\left(\left\{4, m^{*}\right\}\right)$ and $t \geq h$ then $12 t+3 h+m \in B(\{4, m\})$.

Proof (similar to lemma 2, p 282 of [8]): If $v=12 t+3 h+m, K_{v}-K_{m}$ is the disjoint union of four $K_{3 t+m}-K_{m}$, a $K_{3 h+m}-K_{m}$ and the multipartite graph $K_{3 t, 3 t, 3 t, 3 t, 3 h}$. The $K_{3 t+m}-K_{m}$ and $K_{3 h+m}-K_{m}$ can be decomposed into $K_{4}$ 's by hypothesis. As $3 t+m \in B\left(\left\{4, m^{*}\right\}\right)$ then $t \not 2(\bmod 4)$ therefore there exists a transversal design $T(5,1, v)$ (or 3 orthogonal latin squares of order $v$ ) or equivalently a decomposition of $K_{t, t, t, t, t}$ into $K_{5}$ 's. Therefore $K_{t, t, t, t, h}$ for $h \leq t$ can be decomposed into $K_{5}$ 's and $K_{4}$ 's and as $K_{3,3,3,3,3}$ and $K_{3,3,3,3}$ can be decomposed into $K_{4}$ 's, and it follows that $K_{3 t, 3 t, 3 t, 3 t, 3 h}$ can be decomposed into $K_{4}$ 's.

That reduces the determination of $B\left(\left\{4, m^{*}\right\}\right)$ to the verification of the existence of a finite number of $B\left(\left\{4, m^{*}\right\}\right)$ designs. For $m=10$ it suffices to prove the existence of a $B\left(\left\{4, m^{*}\right\}, v\right)$ for $v=31,34,43,46,55,58,67,70,79,82,91,103,118,139,151$, 199.

Lemma 2 (Brouwer, Lemma 7 in [8]): For $t>1,9 t+4 \in B\left(\left\{4,3 t+1^{*}\right\}\right)$.

Here with $t=3$ we have $31 \in B\left(\left\{4,10^{*}\right\}\right)$; with $t=11$ we have $103 \in B\left(\left\{4,34^{*}\right\}\right)$ and therefore to $B\left(\left\{4,10^{*}\right\}\right)$ if $34 \in B\left(\left\{4,10^{*}\right\}\right)$. Similarly with $t=15139 \in B\left(\left\{4,10^{*}\right\}\right)$ if $46 \in B\left(\left\{4,10^{*}\right\}\right)$.

Lemma 3 (similar to Lemma 10 of [8]): If $1 \leq s \leq 4 t+1$ and $3 s+1 \in B\left(\left\{4,10^{*}\right\}\right.$ ), then $36 t+3 s+13 \in B\left(\left\{4,10^{*}\right\}\right)$.

Here

with $s=3, t=1$ we have $58 \in B\left(\left\{4,10^{*}\right\}\right)$

with $s=10, t=3$ we have $151 \in B\left(\left\{4,10^{*}\right\}\right)$

with $s=14, t=4$ we have $199 \in B\left(\left\{4,10^{*}\right\}\right)$.

Lemma 4: If $t+6 \in B\left(\left\{4,7^{*}\right\}\right)$ then $4 t+6 \in B\left(\left\{4,10^{*}\right\}\right)$.

Proof: Let $X=X_{1} \cup X_{2} \cup X_{3} \cup X_{4} \cup Y$ with $|Y|=6$ and $\left|X_{i}\right|=t$. Then the multipartite graph constructed on $X_{1} \cup X_{2} \cup X_{3} \cup X_{4}$ isomorphic to $K_{t, t, t, t}$ can be decomposed into $K_{4}$ 's as 
$t \neq 2,6$. Choose a particular $K_{4},\left\{x_{1}, x_{2}, x_{3}, x_{4}\right\}$ with $x_{i} \in X_{i}$. Then the edges of the $K_{v}$ constructed on $X$ can be partitioned into the four $K_{t+6}-K_{7}$ (each decomposable into $K_{4}$ 's by hypothesis) constructed on $K_{\left\{X_{i} \cup Y\right\}}-K_{\left\{x_{i} \cup Y\right\}}$, the $K_{4}$ 's of $K_{t, t, t, t}$ distinct from $\left\{x_{1}, x_{2}, x_{3}, x_{4}\right\}$ and the $K_{10}$ constructed on $K_{Y \cup\left\{x_{1}, x_{2}, x_{3}, x_{4}\right\}}$.

By choosing

$$
\begin{aligned}
& t+6=22 \text { we have } 70 \in B\left(\left\{4,10^{*}\right\}\right) \\
& t+6=34 \text { we have } 118 \in B\left(\left\{4,10^{*}\right\}\right) .
\end{aligned}
$$

Therefore to prove the theorem it suffices to show the existence of a $B\left(\left\{4,10^{*}\right\}, v\right)$ design for $v=34,43,46,55,67,79,82,91$.

For $v=34$ we will give a decomposition of $K_{24}$ into $K_{4}$ 's and 10 parallel classes of $K_{3}$ 's as follows. Let $X$, the set of vertices of $K_{24}$, be of the form $X=\mathbb{Z}_{6} \times \mathbb{Z}_{4}$; the vertices are labeled $(i, j)$ with $i=0,1, \cdots, 5$ and $j=0,1,2,3$. The decomposition is given below:

Classes of $K_{3}$ 's:

$\begin{array}{lll}\{(0, j),(1, j),(2, j)\} & \{(3, j),(4, j+1),(5, j)\} & \text { for } j=0,1,2,3 \\ \{(0, j),(1, j+1),(3, j)\} & \{(2, j),(4, j),(5, j+1)\} & \text { for } j=0,1,2,3 \\ \{(0, j),(1, j+2),(4, j)\} & \{(2, j),(3, j+2),(5, j)\} & \text { for } j=0,1,2,3 \\ \{(0, j),(1, j+3),(5, j)\} & \{(2, j),(3, j+3),(4, j+2)\} & \text { for } j=0,1,2,3 \\ \{(0, j),(2, j+1),(3, j+1)\} & \{(1, j),(4, j+1),(5, j+3)\} & \text { for } j=0,1,2,3 \\ \{(0, j),(2, j+2),(4, j+1)\} & \{(1, j),(3, j+1),(5, j+2)\} & \text { for } j=0,1,2,3 \\ \{(0, j),(2, j+3),(5, j+1)\} & \{(1, j),(3, j+2),(4, j)\} & \text { for } j=0,1,2,3 \\ \{(0, j),(3, j+2),(4, j+2)\} & \{(1, j),(2, j+1),(5, j)\} & \text { for } j=0,1,2,3 \\ \{(0, j),(3, j+3),(5, j+2)\} & \{(1, j),(2, j+2),(4, j+3)\} & \text { for } j=0,1,2,3 \\ \{(0, j),(4, j+3),(5, j+3)\} & \{(1, j),(2, j+3),(3, j)\} & \text { for } j=0,1,2,3\end{array}$

Class of $K_{4}$ 's:

$\{(i, 0),(i, 1),(i, 2),(i, 3)\}$

for $i=0, \cdots, 5$

For $v=43$ we will give a decomposition of $K_{33}$ into $K_{4}$ 's and 10 parallel classes of $K_{3}$ 's as follows ${ }^{*}$. Let $X$ be the set of vertices of $K_{33}$, of the form

\footnotetext{
* This decomposition is due to Douglas Stinson. In a preliminary version of the paper we gave an erroneous decomposition and we are very indebted for D. Stinson to indicate the error and find the nice following decomposition.
} 
$X=\mathbb{Z}_{6} \times \mathbb{Z}_{5} \cup\left\{a_{0}, a_{1}, a_{2}\right\}$

the vertices of $\mathbb{Z}_{6} \times \mathbb{Z}_{5}$ being labeled $(i, j)$ with $i=0,1,2,3,4,5$ and $j=0,1,2,3,4$. The decomposition is given below:

10 parallel classes of $K_{3}$ 's:

we first have 6 parallel classes $(i=0, \cdots, 5)$, where the $a_{i}$ are taken modulo 3 , each class consisting of the following $11 K_{3}$ 's.

$$
\begin{array}{ll}
\{(i, 1),(i, 3),(i, 4)\} & \{(i+5,1),(i+1,2),(i+2,3)\} \\
\{(i+3,1),(i, 2),(i+4,4)\} & \{(i+2,1),(i+1,3),(i+5,4)\} \\
\left\{(i+1,1),(i+5,3), a_{i+1}\right\} & \left\{(i+4,1),(i+4,5), a_{i}\right\} \\
\left\{(i+3,2),(i+2,5), a_{i+2}\right\} & \{(i+5,2),(i+1,4),(i+3,5)\} \\
\{(i+2,2),(i+4,3),(i+5,5)\} & \{(i+4,2),(i+3,4),(i, 5)\} \\
\{(i+3,3),(i+2,4),(i+1,5)\} &
\end{array}
$$

Then we have 3 parallel classes : $i=0,1,2$.

$$
\begin{array}{ll}
\{(i+1,1),(i+1,2),(i+2,2)\} & \{(i+4,1),(i+4,2),(i+5,2)\} \\
\{(i+2,1),(i, 4),(i+4,5)\} & \{(i+5,1),(i+3,4),(i+1,5)\} \\
\{(i, 1),(i+5,4),(i+5,5)\} & \{(i+3,1),(i+2,4),(i+2,5)\} \\
\{(i, 3),(i+1,3),(i, 5)\} & \{(i+3,3),(i+4,3),(i+3,5)\} \\
\left\{(i, 2),(i+3,2), a_{i}\right\} & \left\{(i+1,4),(i+4,4), a_{i+1}\right\} \\
\left\{(i+2,3),(i+5,3), a_{i+2}\right\} &
\end{array}
$$

Finally the last class consists of $\{(i, j),(i+2, j),(i+4, j)\}$ with $i=0,1$ and $j=0, \cdots, 4$ and $\left\{a_{0}, a_{1}, a_{2}\right\}$.

$K_{4}$ 's (here again the $a_{i}$ are modulo 3 ) :

$$
\begin{array}{ll}
\left\{(i, 1),(i+2,4),(i+3,5), a_{i+1}\right\} & \text { for } i=0, \cdots, 5 \\
\left\{(i, 2),(i, 3),(i+3,4), a_{i+1}\right\} & \text { for } i=0, \cdots, 5 \\
\{(i, 1),(i+1,1),(i+5,2),(i+2,3)\} & \text { for } i=0, \cdots, 5 \\
\{(i, 2),(i+5,3),(i, 4),(i+1,4)\} & \text { for } i=0, \cdots, 5 \\
\{(i, 2),(i+4,3),(i, 5),(i+1,5)\} & \text { for } i=0, \cdots, 5 \\
\{(i, 1),(i+3,1),(i+1,5),(i+4,5)\} & \text { for } i=0,1,2
\end{array}
$$

For $v=46$ we will give a decomposition of $K_{36}$ into 10 parallel classes of $K_{3}$ 's and 5 parallel classes of $K_{4}$ 's as follows. Let $X$ be the set of vertices of $K_{36}$, of the form $X=\mathbb{Z}_{4} \times \mathbb{Z}_{9}$; the vertices are labeled $(i, j)$ with $i=0,1,2,3$ and $j=0,1, \cdots, 8$. 
The decomposition uses the known decomposition of $K_{9}$ into 4 parallel classes of $K_{3}$ 's ([13]). For $t=0,1,2,3$ we form 8 parallel classes by using $K_{3}$ 's on vertices of the type $(i, j) \quad i \neq t$ and use two parallel classes of the $K_{9}$ on the vertices $(t, j)$. The last two parallel classes are formed on the unused classes of the $K_{9}$ 's.

Classes of $K_{3}$ 's:

$\begin{array}{lll}\{(0, j),(1, j+7),(2, j+3)\} & \text { for } j=0, \cdots, 8 \quad \text { and a class from }(3, j) \\ \{(0, j),(1, j+8),(2, j+6)\} & \text { for } j=0, \cdots, 8 \text { and a class from }(3, j) \\ \{(0, j),(1, j+4),(3, j+2)\} & \text { for } j=0, \cdots, 8 \text { and a class from }(2, j) \\ \{(0, j),(1, j+6),(3, j+7)\} & \text { for } j=0, \cdots, 8 \text { and a class from }(2, j) \\ \{(0, j),(2, j+4),(3, j+1)\} & \text { for } j=0, \cdots, 8 \text { and a class from }(1, j) \\ \{(0, j),(2, j+7),(3, j+6)\} & \text { for } j=0, \cdots, 8 \text { and a class from }(1, j) \\ \{(1, j),(2, j+4),(3, j+6)\} & \text { for } j=0, \cdots, 8 \text { and a class from }(2, j) \\ \{(1, j),(2, j+6),(3, j+4)\} & \text { for } j=0, \cdots, 8 \text { and a class from }(2, j) \\ \text { a class from }(i, j) & \text { for } i=0,1,2,3 & \\ \text { a class from }(i, j) & \text { for } i=0,1,2,3 & \end{array}$

Classes of $K_{4}$ 's:

$\begin{array}{ll}\{(0, j),(1, j),(2, j),(3, j)\} & \text { for } j=0, \cdots, 8 \\ \{(0, j),(1, j+1),(2, j+2),(3, j+3)\} & \text { for } j=0, \cdots, 8 \\ \{(0, j),(1, j+2),(2, j+1),(3, j+5)\} & \text { for } j=0, \cdots, 8 \\ \{(0, j),(1, j+3),(2, j+5),(3, j+8)\} & \text { for } j=0, \cdots, 8 \\ \{(0, j),(1, j+5),(2, j+8),(3, j+4)\} & \text { for } j=0, \cdots, 8\end{array}$

For $v=55,67,79,82,91$ we will give a cyclical decomposition of $K_{v-10}$ into $K_{4}$ 's and 10 parallel classes of $K_{3}$ 's as follows. Let $X$ be the set of vertices of $K_{v-10}$, of the form $X=\mathbb{Z}_{v-10}$; the vertices are labeled $i$ with $i=0,1, \cdots, v-11$. The decomposition is given below, where the first $K_{3}$ 's form a parallel class and the other form 3 parallel classes (note that the vertices belong to different congruences modulo 3 ). 
$v=55$

$K_{3}$ 's:

$\{i, i+1, i+5\}$

$\{i, i+2, i+16\}$

$\{i, i+7, i+17\}$

$K_{4}$ 's:

$\{i, i+3, i+12, i+23\} \quad$ for $i=0,1, \cdots, 44$

$\{i, i+6, i+19, i+27\} \quad$ for $i=0,1, \cdots, 44$

for $j=0,1, \cdots, 14$ $\{j, j+15, j+30\}$

$v=67$

$K_{3}$ 's:

$\{j, j+19, j+38\}$

for $j=0,1, \cdots, 18$

$\{i, i+1, i+5\}$

for $i=0,1, \cdots, 56$

$\{i, i+2, i+10\}$

for $i=0,1, \cdots, 56$

$\{i, i+7, i+23\}$

for $i=0,1, \cdots, 56$

$K_{4}$ 's:

$\{i, i+3, i+21, i+33\} \quad$ for $i=0,1, \cdots, 56$

$\{i, i+6, i+17, i+31\} \quad$ for $i=0,1, \cdots, 56$

$\{i, i+9, i+22, i+37\} \quad$ for $i=0,1, \cdots, 56$

$v=82$

$\mathrm{K}_{3}$ 's:

$K_{3}$ 's:

$\{j, j+23, j+46\}$

for $j=0,1, \cdots, 22$

$\{j, j+24, j+48\}$

for $j=0,1, \cdots, 23$

for $i=0,1, \cdots, 68$

$\{i, i+1, i+5\}$

for $i=0,1, \cdots, 71$

$\{i, i+2, i+10\}$

for $i=0,1, \cdots, 68$

$\{i, i+2, i+10\}$

for $i=0,1, \cdots, 71$

for $i=0,1, \cdots, 68$

$\{i, i+7, i+20\}$

for $i=0,1, \cdots, 71$

$K_{4}$ 's:

$\{i, i+18, i+36, i+54\} \quad$ for $i=0,1, \cdots, 17$

$\{i, i+3, i+12, i+41\} \quad$ for $i=0,1, \cdots, 71$

$\{i, i+6, i+28, i+45\} \quad$ for $i=0,1, \cdots, 71$

$\{i, i+11, i+26, i+51\} \quad$ for $i=0,1, \cdots, 71$

$\{i, i+14, i+30, i+49\} \quad$ for $i=0,1, \cdots, 71$ 
$v=91$

$K_{3}$ 's:

$\{j, j+27, j+54\} \quad$ for $j=0,1, \cdots, 26$

$\{i, i+1, i+5\} \quad$ for $i=0,1, \cdots, 80$

$\{i, i+2, i+10\} \quad$ for $i=0,1, \cdots, 80$

$\{i, i+7, i+20\} \quad$ for $i=0,1, \cdots, 80$

$K_{4}$ 's:

$\{i, i+3, i+9, i+37\} \quad$ for $i=0,1, \cdots, 80$

$\{i, i+11, i+33, i+56\} \quad$ for $i=0,1, \cdots, 80$

$\{i, i+12, i+30, i+62\} \quad$ for $i=0,1, \cdots, 80$

$\{i, i+14, i+35, i+52\} \quad$ for $i=0,1, \cdots, 80$

$\{i, i+15, i+39, i+55\} \quad$ for $i=0,1, \cdots, 80$

Acknowledgement: we thank very much A. Hartmann and D. Sotteau for stimulating discussions on this problem. Special thanks are due to D. Stinson who found the decomposition of $K_{43}$ enabling us to finish the proof of the last theorem.

\section{References}

1. A. Assaf, E. Mendelsohn, and D.R. Stinson, "On resolvable coverings of pairs by triples," Utilitas Math., 1987. to appear

2. R.D. Baker and R.M. Wilson, "Nearly Kirkman triple systems," Utilitas Math., vol. 11, pp. 289-296, 1977.

3. C. Berge, Graphs and hypergraphs, North Holland, Amsterdam, 1973.

4. J-C. Bermond, J. Bond, M. Paoli, and C. Peyrat, "Graphs and interconnection Networks: Diameter and Vulnerability," in Surveys in Combinatorics, Proc. of the Ninth British Combinatorial Conference, pp. 1-30, London Mathematical Society, Lecture Note Series 82., 1983.

5. J-C. Bermond, J. Bond, and C. Peyrat, "Bus Interconnection Networks with each station on two buses," in Parallel Algorithms and Architectures, Proc. Int. Coll. Parallel Algorithms and Architectures, Luminy, pp. 155-167, North Holland, 1986.

6. J-C. Bermond, J. Bond, and J-F. Sacle, "Large hypergraphs of diameter 1," in Graph Theory and Combinatorics, Proc. Coll. Cambridge, 1983, pp. 19-28, 
Academic Press, London, 1984.

7. J-C. Bermond, C. Delorme, and J-J. Quisquater, "Strategies for interconnexion networks: Some methods from graph theory,"'Journal of Parallel and Distributed Computing, vol. 3, pp. 433-449, 1986.

8. A.E. Brouwer, "Optimal packings of $K_{4}$ 's into a $K_{n}$," Journal of Comb. Th., ser. $A$, vol. 26, pp. 278-297, 1979.

9. T.Y. Feng, "A Survey of Interconnection Networks," Computers, vol. 14, pp. 12-27, 1981.

10. H. Hanani, "Balanced incomplete block designs and related designs," Discr. Math., vol. 11, pp. 255-363, 1975.

11. C. Huang, E. Mendelsohn, and A. Rosa, "On partially resolvable t-partitions," in Theory and Practice of Combinatorics, Annals of Discrete Mathematics, vol. 12, pp. 169-183, North Holland, Amsterdam, 1982.

12. W. H. Mills, "Covering designs I: Coverings by a small number of subsets," Ars Combinat., vol. 8, pp. 199-315, 1979.

13. D. K. Ray-Chaudhuri and R. M. Wilson, "Solution of Kirkman's schoolgirl problem," in Proc. of Symp. in Pure Math., vol 19 Combinatorics, pp. 187-204, Amer. Math. Soc. Providence, 1971.

14. L.D. Wittie, "Communication Structures for Large Networks of Microcomputers," IEEE Trans. on Computers, vol. C-30, no. 4, pp. 264-273, 1981. 